\title{
Dynamic Thiol/Disulfide Homeostasis In Patients With Newly Diagnosed Type 2 Diabetes Mellitus
}

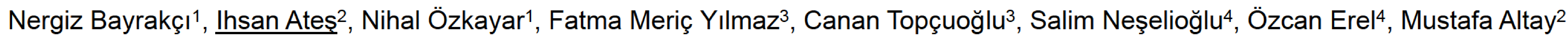

${ }^{1}$ Ankara Numune Education and Research Hospital, Department of Nephrology, Ankara, Turkey

${ }^{2}$ Ankara Numune Education and Research Hospital, Department of Internal Meicine, Ankara, Turkey

${ }^{3}$ Ankara Numune Education and Research Hospital, Department of Biochemistry, Ankara, Turkey

${ }^{4}$ Y 1 ldırım Beyazit University, School of Medicine, Department of Biochemistry, Ankara, Turkey

\section{Objectives:}

\section{Methods:}

\section{Results:}

Conclusions:

\section{References:}

Thiol/disulfide homeostasis plays an important role in antioxidative defense mechanism, detoxification, signal transport, management of enzyme activity and trascription factors, and apoptosis. When thiol/disulphide homeostasis breaks down, these important cellular functions get deranged. Pathologies secondary to oxidative stress are seen in organeles in which this homeostasis is deranged. The aim of the study was to investigate dynamic thiol/disulfide homeostasis in newly diagnosed type 2 diabetes mellitus.

Blood thiol/disulfide homeostasis status, that consists of native thiol-disulphide exchanges, was investigated in 60 patients ( 22 male, 38 female) diagnosed with type 2 diabetes mellitus and 60 healthy control subjects ( 25 male, 35 female). Serum native thiol and total thiol concentrations were measured as a paired test. The half of the difference between total thiol and native thiol concentrations gave the disulphide bond amount.

In comparison to the control group, patients with diabetes mellitus had lower levels of serum thiol and total thiol $(340.7 \pm 46.1 \mu \mathrm{mol} / \mathrm{L}$ vs $313.7 \pm 57.4 \mu \mathrm{mol} / \mathrm{L} ; p=0.005 ; 366.7 \pm 46.7 \mu \mathrm{mol} / \mathrm{L}$ vs $343.7 \pm 59.0$ $\mu \mathrm{mol} / \mathrm{L} ; p=0.020$, respectively) while higher average disulfide level $(12.9 \pm 3.2 \mu \mathrm{mol} / \mathrm{L}$ vs $15.0 \pm 4.6$ $\mu \mathrm{mol} / \mathrm{L} ; \mathrm{p}=0.008)$. In patietns with diabetes mellitus, average disulfide/thiol ratio (\%) $(3.9 \pm 1.2 \mathrm{vs}$ $5.0 \pm 1.6 ; p=0.001)$ and disulfide/total thiol ratio $(\%)(3.6 \pm 1.0$ vs $4.5 \pm 1.6 ; p=0.001)$ was higher while average thiol/total thiol ratio (\%) was lower $(92.8 \pm 2.1 \mathrm{vs} 91.0 \pm 3.4 ; p=0.001)$ than the control group. $\mathrm{HbA} 1 \mathrm{c}$ level and age correlated positively with serum disulfide/thiol ratio and disulfide/total thiol ratio $(r=0.239, p=0.009 ; r=0.228, p=0.012$, respectively $)$ while thiol/total thiol ratio was correlated negatively $(r=-0.228, p=0.012)$.

A tendency towards disulfide formation in thiol/disulfide homeostasis was found in patients with diabetes mellitus and there was a positive correlation between $\mathrm{HbA} 1 \mathrm{c}$ and disulfide/thiol ratio.

1.Erel, O. and S. Neselioglu, A novel and automated assay for thiol/disulphide homeostasis. Clin Biochem, 2014. 47(18): p. 326-32.

2. Rodrigues, S.D., et al., Plasma cysteine/cystine reduction potential correlates with plasma creatinine levels in chronic kidney disease. Blood Purif, 2012. 34(3-4): p. 231-7

3. Ellman, G. and H. Lysko, A precise method for the determination of whole blood and plasma sulfhydryl groups. Anal Biochem, 1979. 93(1): p. 98-102. 